\title{
Research on the Application of ASMR in the Development and Design of Sleeping Products
}

\author{
Miao Wang, Bo Li \\ College of Art and Design,Xi' an University of Technology, ShaanXi, China
}

\begin{abstract}
Sleep is an important basis to keep human's health and physiological activity. But nowadays, sleep disorders, depression, anxiety has become a social problem. Both young and middle-aged people face frequent health problems. In order to improve the sleep quality of the urban population and effectively alleviate the influence of a bad mood before sleep on sleep quality. Through the use of Autonomous Sensory Meridian Response (AMSR) induces a sensory phenomenon to help sleep, combine this new experience with the design, according to the features of this sensory phenomenon formulate a sleeping product scheme, discovering the new way to help to ease symptoms of sleep disorders. Through comparative experiments, test the sleep time of the target population, it is utilized to compare the sleeping time of the subjects after the application of the product. Analyse the feasibility and effectiveness of the product. The result showed that apply this product, $66 \%$ of the subjects reported that it was good to sleep, the validity of this method is proved.
\end{abstract}

\section{Introduction}

With the development of social economy, People's work and study pressure is increasing, the new way of life created many problems, according to the DXY 2019 National Health insight Report, $90 \%$ of citizens don't think their health scores are high, $96 \%$ of the public are not satisfied with their health, the top three problems are poor skin, anxiety, depression and insomnia, but all three points to a conclusion, that's sleep disorders. It is mainly manifested as difficulty in falling asleep or cool to wake up halfway, sleep disorders can lead to lack of energy during the day, lack of concentration, low efficiency of work and learning and another question. According to the survey, $76 \%$ of the people suffer from sleep disturbance. $84 \%$ of the post-90s have trouble sleeping, it is primarily due to poor sleep, difficulty in falling asleep.

Autonomous Sensory Meridian Response (ASMR) was a new type of video that induces relaxation and promotes sleep ${ }^{[1]}$. In recent years, it was very popular on the internet. In 2009, the first channel with whisper content appeared on YouTube. In 2010. Jennifer Allen summarized these phenomena as ASMR. ASMR first appeared in China in 2014, and its promotion was after Luo Zhenyu' s cross year speech on the Internet emerging things in 2016. It will not be long before it enters the public view. For the Chinese market and users, it is also a very small way to help sleep. Some medical researches think that ${ }^{[2]} \mathrm{ASMR}$ can relieve depression, anxiety and chronic pain to some extent.

This paper proposes an innovative idea of combining the new form of ASMR with product design and development, develop and design new products that can help sleep healthily, alleviate sleep problems caused by sleep disorders to achieve comfortable and comfortable sleep assist function.

\section{Research status}

In ASMR video on the network, most of the expression methods are to collect the daily "noise" in life, first, such as the friction of clothes, the knocking sound of daily necessities, the sound of writing and turning over books, which are disordered or some harsh when mixed together and in the daily environment. But when ASMR artists use equipment to make these sounds appear independently in a noiseless video, the sounds in this kind of life can bring emotional comfort to people ${ }^{[3]}$. In most ASMR works, ASMR artists usually appear as a gentle, amiable and intimate character without any aggression, such as doctors, friends, brothers and sisters, or lovers, they play this role to get closer, making it easier for the audience to let go of their guard and relax.

In an experiment conducted by Emma L. Barrat ${ }^{[2]}, 80 \%$ of the participants believed that ASMR had a positive regulatory effect on emotion, $69 \%$ of patients with moderate or severe depression who scored on the BDI (Beck Depression Inventory) said they had alleviated their

$\overline{903622482 @ q q . c o m}$ 
symptoms in this way, in the experiment, patients with depression had significantly more mood boosting effects than those without depression, this shows that ASMR can provide temporary emotional relief for people with depression.

ASMR as a new form of perception, has not a long history, but in some studies, it's a way to relax your mind and calm your mood, Barratt and other researchers even think that ASMR can improve symptoms and relieve chronic pain in patients with depression ${ }^{[4]}$, as a way to relieve anxiety, guiding sleep will be very mild and effective. In the latest hypnotherapy guidelines, it is also recommended to give priority to the use of psychotherapy rather than drugs to help sleep ${ }^{[5]}$.

\section{Application of ASMR in the development and design of hypnotic products}

\subsection{Thoughts on the development of sleep products combined with ASMR}

The Autonomous Sensory Meridian Response (ASMR) is a sensory phenomenon that is typically elicited in response to visual or audio stimuli that cause static, tingling sensations that originate in the head and often spread to the neck and sometimes other regions of the body ${ }^{[2]}$, the audience can hear soothing, soft and smooth sounds, such as whispering, chewing, blowing and so on ${ }^{[6]}$, thus triggering ASMR response.
In general, the most important way to trigger ASMR is through auditory trigger, combined with the analysis results and investigation data, the new therapy can be combined with bedding to improve sleep quality, improve and induce comfortable sleep, among bedding, sleeping pillow is the most closely related to sleep. The 2019 national health survey also showed that $27 \%$ of the people are prepared to buy better sleepers to improve their sleep. The main purpose of this kind of sleep aid product design is to add ASMR playing function to ordinary pillow and turn it into a functional music pillow based on ASMR triggering reaction.

\subsection{Analysis of the components of sleep aid products}

\subsubsection{Material selection. Susan JGordon conducteda series of} experiments to test the pillow materials. The results showed that subjects who used the memory cotton pillow had a certain probability of pain. Depending on the data, the feather pillow will greatly increase the probability of spinal pain after sleeping. If it is employed for a long time, it will easily cause cervical vertebra problems. Latex pillows are the best of all pillow core materials. The subjects who use latex pillows are less likely to have vertebral pain after waking up ${ }^{[7]}$. Therefore, in terms of material selection, latex is the most suitable material for a pillow core. It has excellent elasticity, strong air permeability and enough neck support.

Table.1 Percentage of subjects reporting high pillow comfort and sleep quality ratings

\begin{tabular}{cccccc}
\hline & Polyester (\%) & $\begin{array}{c}\text { Foam regular } \\
(\%)\end{array}$ & $\begin{array}{c}\text { Foam contour } \\
(\%)\end{array}$ & Feather (\%) & Rubber(\%) \\
\hline High comfort rating & 85 & 80 & 71 & 41 & 91.5 \\
High sleep quality Rating & 71 & 72 & 63 & 40 & 81 \\
\hline
\end{tabular}

Origin:SUNAN J.GORDON, KAREN.Pillowuse:the behavior of cervical pain, sleep quality and pillow comfort in side sleepers[J].Manual Therapy, 2009:671-678.

3.2.2. Ergonomic settings. The function of the pillow is to support and protect the human neck and maintain the normal physiological curve of the human cervical spine, so as to promote human sleep, pillows with reasonable design directly affect human health, incorrect sleeping position will cause great damage to human cervical vertebra. Whether the pillow is too high or too low, it will affect the cervical spine, too low pillows are prone to fatigue of the Jugular anterior fascicles and ligaments, too high a pillow can compress the spinal cord, causing degeneration of cervical vertebra patient ${ }^{[8]}$.

According to Dunyao Yan' s research, the change of physiological curvature of the cervical spine is part of the cause of cervical spondylitis. Long term use of pillows with inappropriate height will lead to excessive curvature of the cervical spine, which will stretch and damage the ligaments and joint capsule, cause cervical instability, joint dislocation, and then develop into cervical spondylitis ${ }^{[9]}$.According to Hongjun Li' s ${ }^{[10]}$ Experimental Study on pillow performance and sleep comfort, the most suitable pillow height for sleep is about $6-7 \mathrm{~cm}$ after compression when lying on the back, $7-8 \mathrm{~cm}$ after compression when lying on the side, and 5-6 cm after compression when lying on the front. The length of the pillow should be not less than $50 \mathrm{~cm}$ for side lying people, and not less than $45 \mathrm{~cm}$ for supine and prone people. In terms of pillow width, the pillow width should be greater than $25 \mathrm{~cm}$ in supine, lateral and prone positions. 


\begin{tabular}{ccccccccccc}
\hline $\begin{array}{c}\text { Facial } \\
\text { width } \\
(\mathrm{mm})\end{array}$ & \multicolumn{10}{c}{ morphological facial height $(\mathrm{mm})$} \\
\cline { 2 - 11 } 120 & - & - & - & - & 0.01 & - & - & - & - & - \\
125 & - & 0.02 & 0.13 & 0.42 & 0.50 & 0.22 & 0.08 & 0.03 & - & - \\
130 & 0.01 & 0.14 & 0.75 & 4.44 & 7.15 & 6.24 & 2.33 & 0.52 & 0.04 & - \\
135 & - & 0.04 & 0.82 & 6.51 & 14.80 & 15.75 & 8.54 & 2.16 & 0.29 & 0.04 \\
140 & - & 0.02 & 0.29 & 2.31 & 6.50 & 8.57 & 5.53 & 1.68 & 0.27 & 0.04 \\
145 & - & - & 0.02 & 0.14 & 0.51 & 0.95 & 0.72 & 0.27 & 0.04 & 0.01 \\
150 & - & - & - & - & 0.02 & 0.04 & 0.04 & 0.01 & - & - \\
\hline
\end{tabular}

Fig.1.Two dimensional distribution of face length and width of Chinese adult men

3.2.3. Colour selection. Like shape and material, colour is part of the determinant of product appearance style. Different product demand groups determine different style orientation in colour design, and the colour emotion contained in products will directly affect consumers ${ }^{[11]}$. For bedding, the choice of colour scheme is must use, across all gender and age groups, the usual conservative colour scheme is to choose the cool colour and warm colour respectively for colour matching design.

\section{The design and development principle of ASMR applied to sleep products}

\subsection{Comfort principle}

As an essential support during sleep, pillow plays an important role in people's sleep, unreasonable pillow design and long-term use of pillows with unreasonable structure will lead to changes in the original normal broader, because bedding is a daily necessity that everyone

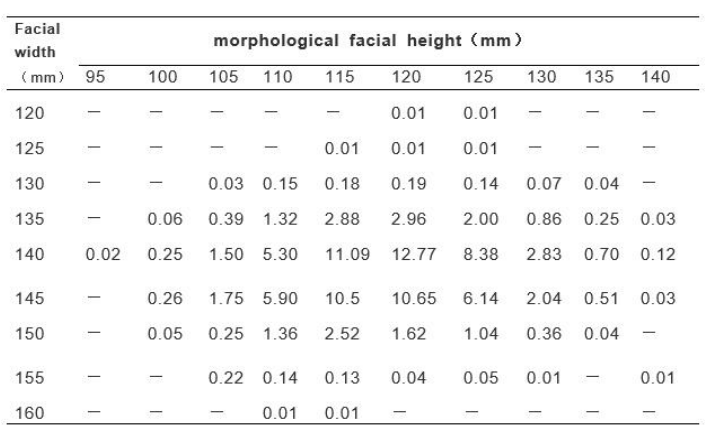

Fig.2. Two dimensional distribution of face length and width of Chinese adult women

physiological curve of the cervical spine, impact on human health. A suitable pillow can greatly improve people's sleep quality, which can be described as one of the determinants of sleep quality ${ }^{[12]}$.

ASMR usually refers to a series of sensory triggering activities including vision, hearing, touch, but in fact, Luciana Besedovsky ${ }^{[13]}$ believe that auditory stimulation is a more direct and effective way to improve sleep quality. Most ASMR listeners also wear headphones to sleep at night. But there are many disadvantages of wearing headphones during sleep, because people act unconsciously when they fall asleep. The change of sleeping posture will lead to the pressure of earphones on the ear canal, which will cause damage and even affect hearing over time. Wearing headphones for a long time will also increase the growth of bacteria in the ear canal. Wired headphones may even be wrapped around the neck and cause suffocation in the process of unconscious rolling during sleeping, which brings a great potential safety hazard.

\subsection{Cost performance principle}

Table. 2 pillows available on the market

\begin{tabular}{cccc}
\hline Brand & Material & Price & Sales volume \\
\hline JOES & polyethylene hose & 139 & $1299 /$ months \\
SNAIL & polyethylene hose & 150 & $2049 /$ months \\
Pilo & Memory Foam & 799 & $16 /$ months \\
AiSleep & Memory Foam & 179 & $331 /$ months \\
POKALEN & Rubber & 299 & $277 /$ months \\
Noyoke & Foam & 149 & $162 /$ months \\
Phealth & Rubber & 276 & $1886 /$ months \\
MUJI & Rubber & 86 & $51 /$ months \\
Benepom & Memory Foam & 1880 & $37 /$ months \\
Cory & Gel & 688 & $143 /$ months \\
\hline
\end{tabular}

Origin:TaoBao.com

Depending on the data in Table 2, most of the prices of slow rebound memory foam pillows are on the high side, of which the most expensive one can reach nearly 2000 yuan, and the sales volume is also the lowest. The price of polyethylene hose is relatively low, and the sales volume is also useful. However, there are only two commodities with this material on the market. The price of rubber pillow is the most moderate, basically in the range of RMB 100-300, and the sales performance is relatively outstanding, it is a good choice for people who can't trust low price goods, but can't afford to consume high price goods.

\subsection{Sustainability}

Under the guidance of ecological philosophy, integrating design behavior into the system of "society environment economy human" is sustainable design ${ }^{[14]}$, which not only 
realizes social value but also protects natural value, when designing products, we should not only consider meeting the needs of customers, but also pay attention to the impact of product use on the environment, for example, using natural materials, recycling materials, environmental protection of product processing technology, avoiding excessive packaging. Nowadays, environmental protection has become a worldwide trend, in 2014. Adidas launched future craft. Loop running shoes. The materials of the whole pair of shoes are made of TPU after recycling the garbage in the ocean, and can be recycled and grinned into raw materials again after being discarded to make new shoes, Stella McCartney, its designer, argues that sustainability is fundamental to design.

\section{Design and development case of sleeping pillow}

The most classical ergonomic constraints are introduced into the design parameters of the sleeping pillow, and the contour design of the sleeping pillow is completed. The pillow adopts the B-type design of up, down and down and the loudspeaker arranged on both sides. There are both low and high occipital areas, visually, the soft pillow core is combined with the awkward speaker shell, with a clear structure, the external speaker is superior to the internal speaker in terms of sound quality and head impact. At the same time, the part of the speaker adopts the honeycomb structure, which is a kind of structure with robust industrial sense, and will bring better visual effect when used in product modeling. The display effect of sleeping pillow products is as shown in the figure.

Loudspeakers are set on both sides of the sleeping pillow. Surround sound can bring to the user an immersion sleep experience. The speaker can be connected to the mobile phone via Bluetooth for ASMR guided sleep.

In ergonomics design of this pillow, the height of the pillow is $10 \mathrm{~cm}$, the height of the pillow is $7 \mathrm{~cm}$, the length of the pillow is $60 \mathrm{~cm}$, and the width is $30 \mathrm{~cm}$. It adopts the form of upper, lower and lower neck support, which conforms to the natural curvature of the cervical spine, lift the vertebrae that need to be lifted up according to the corresponding radian, the back of the head is in a depression, supporting and protecting the cervical vertebra during sleep.
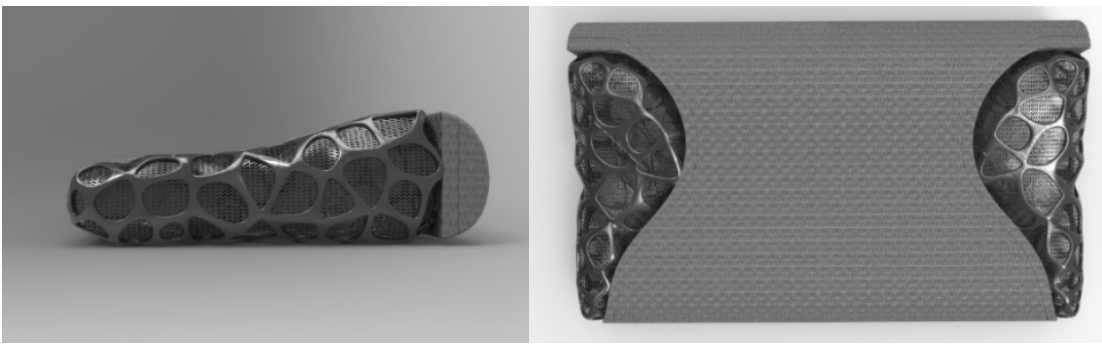

Fig.3-4. Display effect chart of sleeping pillow products

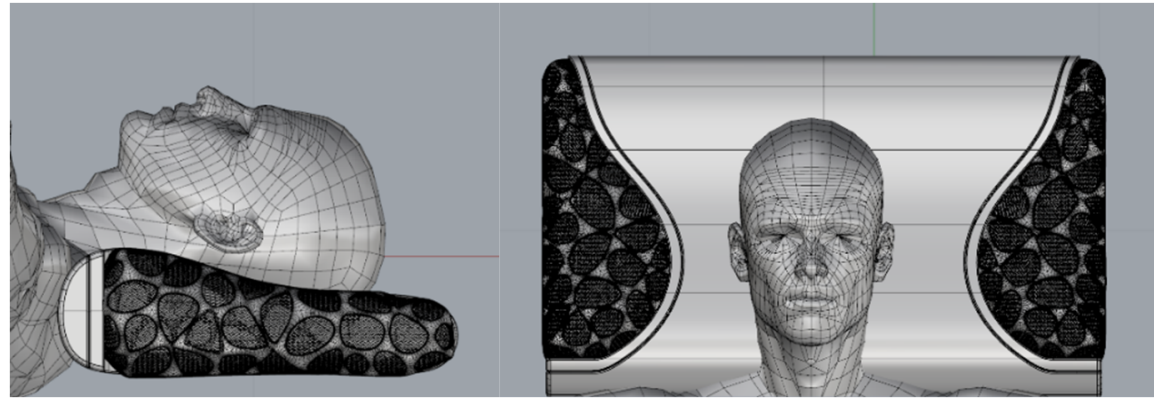

Fig.5-6. Schematic diagram of using the situation of sleeping pillow

\section{Experimental test of the effect of ASMR in the use of hypnotic products}

\subsection{Function setting of sleeping pillow}

Table. 3 Solutions to sleep disorders

\begin{tabular}{cc}
\hline Solutions to sleep disorders (\%) & \\
\hline Regulate sleep time & 50 \\
Use more comfortable Pillows / mattresses & 27 \\
Adjust diet & 17 \\
Use earmuffs / earplugs & 15 \\
Health products & 4 \\
Aromatherapy & 3 \\
Hypnotic drugs & 3 \\
Others & 3 \\
Noting & 27 \\
\hline
\end{tabular}


Origin: 2019 National Health insight Report In the data in Table 3, 27\% of sleep impaired people are willing to improve their sleep by choosing a better pillow mattress, in the process of sleep, pillow is also one of the indispensable necessities for people, which plays an important role in the quality of sleep, pillows of correct material and shape can provide good support and protection for cervical vertebra and head.

According to the above analysis, a pillow product with ASMR function is developed based on the characteristics of spontaneous perception meridians system and the pillow.

\subsection{Experimental sample and process}

This survey includes 8 people born in 1970s-1980s, 10 people born in 90 generations, $20 \%$ of them have anxiety or depression tendency and sleep disorder trend in different degrees. Two people have a history of pathological insomnia, a total of 18 subjects were tested for one week(7days) sleep monitoring.

The experiment was conducted in a quiet environment, subjects were asked to give daily feedback on the sleep experience of the second day after the previous night's use of sleep aid products, it includes whether it can relieve the mood before falling asleep, the time of falling asleep, the time of entering deep sleep, the duration of sleep, the degree of brain wakefulness after waking up in the morning, and whether there are any adverse reactions after waking up, or whether there is any difference between waking up and normal comparison. Subjects were asked to present a comparative vocabulary after waking up on the fourth day and the first day, subjects were asked to compare their feelings on the morning of the eighth day, at the end of the experiment, with those on the first day of the experiment.

Table.4 General information about subjects

\begin{tabular}{ccccc}
\hline Age Bracket & $19-29$ & $30-39$ & $40-49$ & Total \\
\hline Number & 10 & 3 & 5 & 18 \\
Proportion & $55.6 \%$ & $16.7 \%$ & $27.7 \%$ & \\
\hline
\end{tabular}

Table.5 Comparison of sleep duration of subjects

\begin{tabular}{ccccccccc}
\hline & \multicolumn{2}{c}{$<5 \mathrm{~h}$} & \multicolumn{2}{c}{$5-6 \mathrm{~h}$} & \multicolumn{2}{c}{$7-8 \mathrm{~h}$} & \multicolumn{2}{c}{$>9 \mathrm{~h}$} \\
\hline & cases & ratio & cases & ratio & cases & ratio & cases & ratio \\
Day1 & 5 & $27.8 \%$ & 9 & $50 \%$ & 4 & $22.3 \%$ & 0 & $0 \%$ \\
Day4 & 2 & $11 \%$ & 10 & $55.6 \%$ & 4 & $22.3 \%$ & 2 & $11 \%$ \\
Day7 & 0 & $0 \%$ & 6 & $33.3 \%$ & 9 & $50 \%$ & 2 & $11 \%$ \\
\hline
\end{tabular}

Result: after 7 days, fewer subjects slept less than five hours, people who sleep 5 to 6 hours after 7 days have less, the proportion of people in 7-8 hours increased to $50 \%$, sleep time of more than 5 hours increased to $11 \%$.

Table. 6 Comparison of falling asleep duration of subjects

\begin{tabular}{ccccccccc}
\hline \multicolumn{2}{c}{$<10 \mathrm{~min}$} & \multicolumn{2}{c}{$10-30 \mathrm{~min}$} & \multicolumn{2}{c}{$30-60 \mathrm{~min}$} & \multicolumn{2}{c}{$>1 \mathrm{~h}$} \\
\hline & cases & ratio & cases & ratio & cases & ratio & cases & ratio \\
Day1 & 4 & $22.3 \%$ & 6 & $33.3 \%$ & 4 & $22.3 \%$ & 2 & $11 \%$ \\
Day4 & 10 & $55.65 \%$ & 6 & $33.3 \%$ & 2 & $11 \%$ & 0 & $0 \%$ \\
Day7 & 12 & $66.7 \%$ & 6 & $33.3 \%$ & 0 & $0 \%$ & 0 & $0 \%$ \\
\hline
\end{tabular}

Result: after 7 days, $66.7 \%$ of the subjects slept more than 10 minutes, fewer people fall asleep for more than 30 minutes.

Table.7 Sleep state comparisons

\begin{tabular}{ccccccccc}
\hline & \multicolumn{2}{c}{ Unable to sleep well } & \multicolumn{2}{c}{ Sleep till dawn } & \multicolumn{2}{c}{ Wake up feeling sick } & \multicolumn{2}{c}{ More Dream } \\
\hline & cases over times $>10$ & ratio & cases & ratio & cases & ratio & cases & ratio \\
Day1 & 10 & $55.6 \%$ & 5 & $27.8 \%$ & 7 & $38.9 \%$ & 13 & $72.2 \%$ \\
Day4 & 6 & $33.35 \%$ & 8 & $44.5 \%$ & 5 & $27.7 \%$ & 10 & $55.6 \%$ \\
Day7 & 2 & $11 \%$ & 16 & $88.9 \%$ & 2 & $11 \%$ & 4 & $22.3 \%$ \\
\hline
\end{tabular}

Result: after 7 days, the number of people who reported that they could not sleep steadily decreased from $55.6 \%$ to $11 \%$, the number of people who slept until dawn increased from $27.8 \%$ to $88.9 \%$, feedback wakes up discomfort from $38.8 \%$ to $11 \%$, the number of people who reported having more dreams decreased from $72.2 \%$ to $22.3 \%$.

After 7 days, the sleep state of the subjects improved to a certain extent, which proved the effectiveness of this method.

\section{Conclusion}

ASMR as a new way of sleeping, although it hasn't been long enough to get into the public eye, some studies have proved that this is an effective adjuvant therapy for sleep and mental relaxation, through this trigger mechanism, it can help users better induce sleep and relieve emotions, so as to relieve the pressure in life and sleep better. With good sleep, you can have sufficient spirit to deal with the next 
day's work and life. In this study, we propose a method to design and develop a sleeping pillow, which can induce sleep and improve sleep quality by using the sensory response caused by autonomous sensory meridian response and the corresponding sleeping products, it has definite promotion value.

\section{Reference}

1. Liu M, Zhou Q. A Preliminary Compilation of a Digital Video Library on Triggering Autonomous Sensory Meridian Response (ASMR): A Trial Among 807 Chinese College Students. Front Psychol. 2019;10:2274. Published 2019 Oct 15. doi:10.3389/fpsyg.2019.02274

2. Barratt EL, Davis NJ.Autonomous Sensory Meridian Response (ASMR): a flow-like mentalstate.PeerJ. 2015;3:e851. Published 2015 Mar 26. doi: $10.7717 /$ peerj.851

3. Naomi Smith,Anne-Marie Snider. ASMR, affect and digitally-mediated intimacy[J]. Emotion, Space and Society, 2018.

4. Barratt EL, Spence C, Davis NJ. Sensory determinants of the autonomous sensory meridian response (ASMR):understandingthe triggers. PeerJ. 2017;5:e3846. Published 2017 Oct 6.

5. Thorpy M.International Classification of Sleep Disorders[M]. Sleep Disorders Medicine.New York: Springer,2017: $475-484$

6. Lloyd J V , Ashdown T O , Jawad L $\mathrm{R}$. Autonomous Sensory MeridianResponse: What is It and Why Should We Care[J].Indian J PsycholMed,2017,39(2) : 214-215

7. SUNAN J.GORDON, KAREN.Pillowuse:the behavior ofcervical pain, sleepquality and pillow comfort in side sleepers[J].Manual Therapy, 2009:671-678

8. CuijuanSu, Guangwu Sun. Suggestions on the scientific use of pillows to prevent and treat cervical spondylosis $[\mathrm{J}]$. Chinese Journal of Orthopaedic Surgery, 2002, 10 (11): 1143

9. Dunyao Yan. Preliminary study on the relationship between pillow and shoulder spleen levator injury [J]. Neck and back pain, 1997,18 (4): 245

10. Hongjun Li. Influence of filler selection on compression recovery performance of pillow core $[\mathrm{J}]$ Jiangsu textile, 2014 (09): 44-46 + 51

11. Wenyu Wu, Tianjian Cui, Shen Zheng.Emotional expression of product color $[\mathrm{J}]$. Packaging engineering, 2010,31 (16): 42-44

12. Min Wang, Jun Li. Application of ergonomics in pillow design $[\mathrm{J}]$. China personal protective equipment, 2008 (05): 19-21

13. Besedovsky L, Ngo HV, Dimitrov S, Gassenmaier C, Lehmann R, Born J. Auditory closed-loop stimulation of EEG slow oscillations strengthens sleep and signs of its immune-supportive function. Nat Commun. 2017;8(1):1984. Published 2017 Dec 7.

14. Jianguo Yin, Zhijun $\mathrm{Wu}$, Chengai Na. Elements and methods of product sustainable design [J]. Journal of Hunan University of science and Technology (NATURAL SCIENCE EDITION), 2018,33 (03): 65-68 